\title{
EQUIDISTRIBUTION OF EXPANDING CURVES IN HOMOGENEOUS SPACES AND DIOPHANTINE APPROXIMATION ON SQUARE MATRICES
}

\author{
LEI YANG
}

(Communicated by Nimish Shah)

\begin{abstract}
In this paper, we study an analytic curve $\varphi: I=[a, b] \rightarrow$ $\mathrm{M}(n \times n, \mathbb{R})$ in the space of $n$ by $n$ real matrices. There is a natural map $u: \mathrm{M}(n \times n, \mathbb{R}) \rightarrow H=\mathrm{SL}(2 n, \mathbb{R})$. Let $G$ be a Lie group containing $H$ and $\Gamma<G$ be a lattice of $G$. Let $X=G / \Gamma$. Then given a dense $H$-orbit in $X$, one could embed $u(\varphi(I))$ into $X$. We consider the expanding translates of the curve by some diagonal subgroup $A=\{a(t): t \in \mathbb{R}\} \subset H$. We will prove that if $\varphi$ satisfies certain geometric conditions, then the expanding translates will tend to be equidistributed in $G / \Gamma$, as $t \rightarrow+\infty$. As an application, we show that for almost every point on $\varphi(I)$, the Diophantine approximation given by Dirichlet's Theorem is not improvable.
\end{abstract}

\section{INTRODUCTION}

1.1. Dirichlet's Theorem on Diophantine approximation. For any real vector space $V=\mathbb{R}^{k}$, let $\|\cdot\|$ denote the supreme norm on $V$, i.e., for $\mathbf{x}=\left(x_{1}, x_{2}, \ldots, x_{k}\right)$ $\in \mathbb{R}^{k},\|\mathbf{x}\|:=\max _{1 \leq i \leq k}\left|x_{i}\right|$. Given $m, n \in \mathbb{N}$, let $\mathrm{M}(m \times n, \mathbb{R})$ denote the space of $m$ by $n$ real matrices. The Dirichlet's Theorem on Diophantine properties of $m$ by $n$ matrices says the following:

Theorem 1.1 (Dirichlet's Theorem). Given any $\Phi \in \mathrm{M}(m \times n, \mathbb{R})$ and any $N \geq 1$, there exists a nonzero integer vector $\mathbf{p} \in \mathbb{Z}^{n}$ with $\|\mathbf{p}\| \leq N^{m}$ and an integer vector $\mathbf{q} \in \mathbb{Z}^{m}$ such that $\|\Phi \mathbf{p}-\mathbf{q}\| \leq N^{-n}$.

Now we consider the following finer question: for a particular $\Phi \in \mathrm{M}(m \times n, \mathbb{R})$, could we improve Dirichlet's Theorem? By improving Dirichlet's Theorem, we mean that there exists a constant $0<\mu<1$, such that for all large $N>0$, there exists a nonzero integer vector $\mathbf{p} \in \mathbb{Z}^{n}$ with $\|\mathbf{p}\| \leq \mu N^{m}$, and an integer vector $\mathbf{q} \in \mathbb{Z}^{m}$ such that $\|\Phi \mathbf{p}-\mathbf{q}\| \leq \mu N^{-n}$. If such constant $\mu$ exists, then we say that $\Phi$ is $\mathrm{DT}_{\mu}$-improvable. We say that $\Phi$ is DT-improvable if $\Phi$ is $\mathrm{DT}_{\mu}$-improvable for some $0<\mu<1$ (here DT stands for Dirichlet's Theorem).

In 1970, Davenport and Schmidt DS70, considered this question and answered it to some extent: they proved that almost every matrix $\Phi \in \mathrm{M}(m \times n, \mathbb{R})$ is

Received by the editors August 11, 2015 and, in revised form, February 19, 2016, February 23, 2016 and February 24, 2016.

2010 Mathematics Subject Classification. Primary 37A17; Secondary 22F30, 11J13.

Key words and phrases. Equidistribution, homogeneous spaces, Ratner's Theorem, Diophantine approximation, Dirichlet's Theorem.

The author was supported in part by a Postdoctoral Fellowship at MSRI. 
not DT-improvable. In [DS70], it was also proved that for $m=1$ and $n=2$, $\mathrm{M}(1 \times 2, \mathbb{R})=\mathbb{R}^{2}$, almost every point on the curve

$$
\phi(s)=\left(s, s^{2}\right): s \in \mathbb{R}
$$

is not $\mathrm{DT}_{1 / 4}$-improvable. Their result on the curve $\phi$ was generalized by Baker Bak78]: it was proved that for any smooth curve in $\mathbb{R}^{2}$ satisfying some curvature condition, almost every point on the curve is not $\mathrm{DT}_{\mu}$-improvable for some $0<$ $\mu<1$ depending on the curve. In 2002, Bugeaud Bug02 generalized the result of Davenport and Schmidt in the following sense: for $m=1$, and general $n$, almost every point on the curve $\varphi(s)=\left(s, s^{2}, \ldots, s^{n}\right)$ is not $\mathrm{DT}_{\mu}$-improvable for some small constant $0<\mu<1$. Their proofs are based on the technique of regular systems introduced by Davenport and Schmidt DS70.

Based on the work of Dani Dan84, as well as Kleinbock and Margulis [KM98], Kleinbock and Weiss KW08 studied this Diophantine approximation problem in the language of homogeneous dynamics, and proved the following result: for $m=1$ and arbitrary $n$, if an analytic curve in $\mathrm{M}(1 \times n, \mathbb{R}) \cong \mathbb{R}^{n}$ satisfies some nondegeneracy condition, then almost every point on the curve is not $\mathrm{DT}_{\mu}$-improvable for some small constant $0<\mu<1$ depending on the curve. In 2009, Nimish Shah Sha09b] proved the following stronger result: for $m=1$ and general $n$, if an analytic curve $\varphi: I=[a, b] \rightarrow \mathbb{R}^{n}$ is not contained in any proper affine subspace, then almost every point on the curve is not DT-improvable.

In this paper, we focus on the case of square matrices. We will prove the following theorem.

Theorem 1.2. For $m=n$, suppose an analytic curve

$$
\varphi: I=[a, b] \rightarrow \mathrm{M}(n \times n, \mathbb{R})
$$

satisfies the following conditions:

A.1 For some $s_{0} \in I$ the derivative $\varphi^{(1)}\left(s_{0}\right)$ is invertible (in particular, this implies that there exists a closed subinterval $J_{s_{0}} \subset I$ such that $\varphi(s)-\varphi\left(s_{0}\right) \in$ $\mathrm{GL}(n, \mathbb{R})$ for $\left.s \in J_{s_{0}}\right)$.

A.2 There exists a $s_{0} \in I$ satisfying the condition above such that $\{(\varphi(s)-$ $\left.\left.\varphi\left(s_{0}\right)\right)^{-1}: s \in J_{s_{0}}\right\}$ is not contained in any proper affine subspace of $\mathrm{M}(n \times n, \mathbb{R})$.

Then almost every point on the curve is not DT-improvable.

Remark 1.3. Because $\varphi$ is analytic, $\varphi^{(1)}(s)$ is invertible at some $s_{0} \in I$ implies that it is invertible for all $s \in I$ except finitely many points.

1.2. Equidistribution of expanding curves on homogeneous spaces. Now we describe the correspondence between Diophantine approximation and homogeneous dynamics.

Let $G=\mathrm{SL}(m+n, \mathbb{R})$, and let $\Gamma=\mathrm{SL}(m+n, \mathbb{Z})$. The homogeneous space $X=G / \Gamma$ may be identified with the space of unimodular lattices of $\mathbb{R}^{m+n}$. In this identification, $g \Gamma$ corresponds to the unimodular lattice $g \mathbb{Z}^{m+n}$. For $r>0$, let $B_{r}$ denote the closed ball in $\mathbb{R}^{m+n}$ centered at the origin and of radius $r$. For any $0<\mu<1$, the subset

$$
K_{\mu}:=\left\{\Lambda \in G / \Gamma: \Lambda \cap B_{\mu}=\{\mathbf{0}\}\right\}
$$


contains an open neighborhood of $\mathbb{Z}^{m+n} \in X$. Define

$$
A:=\left\{a(t):=\left[\begin{array}{ll}
e^{n t} \mathrm{I}_{m} & \\
& e^{-m t} \mathrm{I}_{n}
\end{array}\right]: t \in \mathbb{R}\right\} .
$$

Let us define the embedding $u: \mathrm{M}(m \times n, \mathbb{R}) \hookrightarrow \mathrm{SL}(m+n, \mathbb{R})$ by

$$
\Phi \in \mathrm{M}(m \times n, \mathbb{R}) \mapsto u(\Phi):=\left[\begin{array}{cc}
\mathrm{I}_{m} & \Phi \\
& \mathrm{I}_{n}
\end{array}\right] .
$$

Suppose $\Phi \in \mathrm{M}(m \times n, \mathbb{R})$ is $\mathrm{DT}_{\mu}$-improvable for some $0<\mu<1$; then for any $N>0$ large enough, there exist a nonzero integer vector $\mathbf{p} \in \mathbb{Z}^{n}$ and an integer vector $\mathbf{q} \in \mathbb{Z}^{m}$, such that $\|\mathbf{p}\| \leq \mu N^{m}$ and $\|\Phi \mathbf{p}-\mathbf{q}\| \leq \mu N^{-n}$. Then we have that $\|a(\log N) u(\Phi)(-\mathbf{q}, \mathbf{p})\| \leq \mu$. This implies that $a(\log N) u(\Phi) \mathbb{Z}^{m+n} \notin K_{\mu}$ for all $N>0$ large enough. Thus, to prove that $\Phi \in \mathrm{M}(m \times n, \mathbb{R})$ is not $\mathrm{DT}_{\mu}$-improvable, it suffices to show that the forward trajectory $\{a(t) u(\Phi)[e]: t>0\}$ meets $K_{\mu}$ infinitely many times. In particular, for an analytic curve

$$
\varphi: I=[a, b] \rightarrow \mathrm{M}(m \times n, \mathbb{R}),
$$

if we could show that the expanding translates $\{a(t) u(\varphi(I))[e]: t>0\}$ tend to be equidistributed in $X$, then we could deduce that for almost every point $\varphi(s)$ on the curve, the forward trajectory $\{a(t) u(\varphi(s))[e]: t>0\}$ is dense (see [Sha09b, §2] for the proof). This implies that almost every $\varphi(s)$ is not DT-improvable.

It turns out that when $m=n$, we could prove the following much stronger result:

Theorem 1.4. Let $G$ be a Lie group containing $H=\operatorname{SL}(2 n, \mathbb{R})$, and $\Gamma<G$ be a lattice in $G$. Let $\mu_{G}$ denote the unique $G$-invariant probability measure on the homogeneous space $G / \Gamma$. Take $x=g \Gamma \in G / \Gamma$ such that its $H$-orbit $H x$ is dense in $G / \Gamma$. Let us fix the diagonal group

$$
A=\left\{a(t)=\left[\begin{array}{ll}
e^{t} \mathrm{I}_{n} & \\
& e^{-t} \mathrm{I}_{n}
\end{array}\right]\right\}
$$

Let $\varphi: I=[a, b] \rightarrow \mathrm{M}(n \times n, \mathbb{R})$ be an analytic curve, and embed the curve into $H$ via

$$
u: X \in \mathrm{M}(n \times n, \mathbb{R}) \mapsto u(X)=\left[\begin{array}{ll}
\mathrm{I}_{n} & X \\
& \mathrm{I}_{n}
\end{array}\right] .
$$

Let $\mu_{t}$ denote the normalized Lebesgue measure on the curve $a(t) u(\varphi(I)) x \subset G / \Gamma$, i.e., for a compactly supported continuous function $f \in C_{c}(G / \Gamma)$,

$$
\int f \mathrm{~d} \mu_{t}:=\frac{1}{|I|} \int_{s \in I} f(a(t) u(\varphi(s)) x) \mathrm{d} s .
$$

If the curve $\varphi$ satisfies the conditions $\mathbf{\mathbf { A . 1 }}$ and $\mathbf{\mathbf { A . 2 }}$ given in Theorem $\mathbf{1 . 2}$, then $\mu_{t} \rightarrow \mu_{G}$ as $t \rightarrow+\infty$ in weak-* topology, i.e., for any function $f \in C_{c}(G / \Gamma)$,

$$
\lim _{t \rightarrow+\infty} \frac{1}{|I|} \int_{s \in I} f(a(t) u(\varphi(s)) x) \mathrm{d} s=\int_{G / \Gamma} f \mathrm{~d} \mu_{G} .
$$

Remark 1.5.

1. The assumption that $H x$ is dense in $G / \Gamma$ does not reduce the generality of the theorem. In fact, since $H$ is generated by unipotent subgroups contained in $H$, by Ratner's Theorem (cf. Rat91b]), the closure of $H x$ must be some homogeneous subspace $F x$ where $F$ is some Lie subgroup of $G$ containing $H$ such that the orbit $F x$ is closed and of finite volume. Then we can make the assumption hold by replacing $G, \Gamma$ and $x=g \Gamma$ by $F, g \Gamma g^{-1} \cap F$ and [e] respectively. 
2. To ensure the equidistribution, in condition $\mathbf{A . 2}$ given in Theorem 1.2, we only need $\left\{\left(\varphi(s)-\varphi\left(s_{0}\right)\right)^{-1}: s \in J_{s_{0}}\right\}$ to avoid a family of special proper affine subspaces coming from a particular representation of $H$. We will discuss it in Remark 4.4

3. Even in the case $G=H=\mathrm{SL}(2 n, \mathbb{R})$, Theorem 1.4 is much stronger than Theorem 1.2 since it applies to arbitrary lattice $\Gamma \subset G$.

1.3. Relation to extremity of submanifolds in matrix spaces. Another direction to study Diophantine properties of a real matrix $\Phi \in \mathrm{M}(m \times n, \mathbb{R})$ is to determine whether $\Phi$ is very well approximable. We say $\Phi \in \mathrm{M}(m \times n, \mathbb{R})$ is very well approximable if there exists some constant $\delta>0$ such that there exist infinitely many nonzero integer vectors $\mathbf{p} \in \mathbb{Z}^{n}$ and integer vectors $\mathbf{q} \in \mathbb{Z}^{m}$ such that

$$
\|\Phi \mathbf{p}-\mathbf{q}\| \leq\|\mathbf{p}\|^{-n / m-\delta} .
$$

A submanifold $\mathcal{U} \subset \mathrm{M}(m \times n, \mathbb{R})$ is called extremal if with respect to the Lebesgue measure on $\mathcal{U}$, almost every point is not very well approximable. Given a submanifold in $\mathrm{M}(m \times n, \mathbb{R})$, it is natural to ask whether it is extremal. Because of the same correspondence as above, this problem can also be studied through homogenous dynamics. Kleinbock and Margulis KM98 proved that if a submanifold $\mathcal{U} \subset \mathrm{M}(1 \times n, \mathbb{R})=\mathbb{R}^{n}$ is nondegenerate, then $\mathcal{U}$ is extremal. Kleinbock, Margulis and Wang KMW10 gave a necessary and sufficient condition of a submanifold in $\mathrm{M}(m \times n, \mathbb{R})$ being extremal. The condition is stated in terms of a particular representation of $H=\mathrm{SL}(m+n, \mathbb{R})$ and could not be translated to a geometric condition. Recently, Aka, Breuillard, Rosenzweig and de Saxcé ABRdS14 defined a family of proper subvarieties in $\mathrm{M}(m \times n, \mathbb{R})$ called pencils, and announced a theorem stating that if a submanifold $\mathcal{U} \subset \mathrm{M}(m \times n, \mathbb{R})$ is not contained in a pencil, then $\mathcal{U}$ is extremal. It turns out that condition $\mathbf{A . 1}$ in Theorem 1.2 implies the sufficient condition given in ABRdS14. We will discuss it in detail in Appendix A.

1.4. Organization of the paper. The paper is organized as follows. In $\S 2$, we will follow the argument developed in Sha09c] to modify $\mu_{t}$ to another probability measure $\lambda_{t}$ for each $t>0$. We will show that $\lambda_{t} \rightarrow \mu_{G}$ will imply $\mu_{t} \rightarrow \mu_{G}$ and that any limit measure $\mu_{\infty}$ of $\left\{\lambda_{t}: t>0\right\}$ is a probability measure and is invarant under the action of a unipotent subgroup. In $\S 3$, we will apply Ratner's Theorem and the linearization technique to show that if the limit measure $\mu_{\infty}$ is not $\mu_{G}$, then the curve $\varphi(I)$ must satisfy some linear algebraic condition with respect to a particular finite dimensional representation $\mathcal{V}$ of $H=\mathrm{SL}(2 n, \mathbb{R})$. In $\S 4$, we will complete the proof of Theorem 1.4. The proof is based on the linear algebraic condition we get in $\S 3$, and a technical lemma proved in Yan13 on $\mathrm{SL}(2, \mathbb{R})$ representations. In Appendix A we will discuss the condition given in ABRdS14 and its relation to condition $\mathbf{A . 1}$ in Theorem 1.2 ,

1.5. Notation. Throughout this paper, we will use the following notation.

Let $\pi: G \rightarrow X=G / \Gamma$ denote the natural projection mapping $g \in G$ to $g \Gamma \in X$.

For $\epsilon>0$ small, and two quantities $A$ and $B, A \stackrel{\epsilon}{\approx} B$ means that $|A-B| \leq \epsilon$. Fix a right $G$-invariant metric $d(\cdot, \cdot)$ on $G$; then for $x_{1}, x_{2} \in G / \Gamma$, and $\epsilon>0, x_{1} \stackrel{\epsilon}{\approx} x_{2}$ means $x_{2}=g x_{1}$ such that $d(g, e)<\epsilon$. Given some quantity $A>0$, we denote by $O(A)$ some quantity $B$ such that $|B| \leq C A$ for some constant $C>0$. 
Let $F$ be a Lie group, and $V$ be a finite dimensional linear representation of $F$. Then for a one-parameter diagonal subgroup $A=\{a(t): t \in \mathbb{R}\}$ of $F$, we could decompose $V$ as a direct sum of eigenspaces of $A$, i.e.,

$$
V=\bigoplus_{\lambda \in \mathbb{R}} V^{\lambda}(A)
$$

where $V^{\lambda}(A)=\left\{v \in V: a(t) v=e^{\lambda t} v\right\}$.

We define

$$
\begin{aligned}
& V^{+}(A)=\bigoplus_{\lambda>0} V^{\lambda}(A), \\
& V^{-}(A)=\bigoplus_{\lambda<0} V^{\lambda}(A),
\end{aligned}
$$

and similarly,

$$
\begin{aligned}
& V^{+0}(A)=V^{+}(A)+V^{0}(A), \\
& V^{-0}(A)=V^{-}(A)+V^{0}(A) .
\end{aligned}
$$

For a vector $v \in V$, we denote by $v^{+}(A)\left(v^{-}(A), v^{0}(A), v^{+0}(A)\right.$ and $v^{-0}(A)$ respectively) the projection of $v$ onto $V^{+}(A)\left(V^{-}(A), V^{0}(A), V^{+0}(A)\right.$ and $V^{-0}(A)$ respectively).

\section{NONDIVERGENCE OF LIMIT MEASURES AND UNIPOTENT INVARIANCE}

2.1. Preliminaries on Lie group structures. We first recall some basic facts on $H=\mathrm{SL}(2 n, \mathbb{R})$.

Let $A \subset H$ denote the one-parameter diagonal subgroup defined in (1.1), and let $Z_{H}(A)$ denote the centralizer of $A$ in $H$. It is easy to see that

$$
Z_{H}(A)=\left\{\left[\begin{array}{ll}
B & \\
& C
\end{array}\right]: B, C \in \mathrm{GL}(n, \mathbb{R}), \text { and } \operatorname{det} B \operatorname{det} C=1\right\} .
$$

Define

$$
U^{+}(A):=\{h \in H: a(-t) h a(t) \rightarrow e \text { as } t \rightarrow+\infty\},
$$

and

$$
U^{-}(A):=\{h \in H: a(t) h a(-t) \rightarrow e \text { as } t \rightarrow+\infty\} .
$$

Then it is easy to see that $U^{+}(A)$ and $U^{-}(A)$ can be parametrized as follows:

$$
U^{+}(A)=\left\{u(X)=\left[\begin{array}{ll}
\mathrm{I}_{n} & X \\
& \mathrm{I}_{n}
\end{array}\right]: X \in \mathrm{M}(n \times n, \mathbb{R})\right\},
$$

and

$$
U^{-}(A)=\left\{u^{-}(X)=\left[\begin{array}{cc}
\mathrm{I}_{n} & \\
X & \mathrm{I}_{n}
\end{array}\right]: X \in \mathrm{M}(n \times n, \mathbb{R})\right\} .
$$

The Lie algebras $\mathfrak{u}^{ \pm}(A)$ of $U^{ \pm}(A)$ can be parametrized as follows:

$$
\mathfrak{u}^{+}(A)=\left\{\mathfrak{n}^{+}(X):=\log u(X)=\left[\begin{array}{cc}
\mathbf{0} & X \\
\mathbf{0} & \mathbf{0}
\end{array}\right]: X \in \mathrm{M}(n \times n, \mathbb{R})\right\},
$$

and

$$
\mathfrak{u}^{-}(A)=\left\{\mathfrak{n}^{-}(X):=\log u^{-}(X)=\left[\begin{array}{ll}
\mathbf{0} & \mathbf{0} \\
X & \mathbf{0}
\end{array}\right]: X \in \mathrm{M}(n \times n, \mathbb{R})\right\} .
$$

It is easy to see that $Z_{H}(A)$ normalizes $U^{+}(A)$ and $U^{-}(A)$. In fact, for

$$
z=\left[\begin{array}{ll}
B & \\
& C
\end{array}\right] \in Z_{H}(A),
$$


we have that

$$
\operatorname{Ad}_{z}\left(\mathfrak{n}^{+}(X)\right)=\mathfrak{n}^{+}\left(B X C^{-1}\right)
$$

and

$$
\operatorname{Ad}_{z}\left(\mathfrak{n}^{-}(X)\right)=\mathfrak{n}^{-}\left(C X B^{-1}\right) .
$$

The above two equations give two different actions of $Z_{H}(A)$ on $\mathrm{M}(n \times n, \mathbb{R})$. Let us denote them by $\mathrm{Ad}^{+}$and $\mathrm{Ad}^{-}$, respectively.

Define

$$
\mathfrak{a}:=\left[\begin{array}{cc}
\mathrm{I}_{n} & \mathbf{0} \\
\mathbf{0} & -\mathrm{I}_{n}
\end{array}\right]
$$

For any $X \in \operatorname{GL}(n, \mathbb{R}),\left\{\mathfrak{n}^{+}(X), \mathfrak{n}^{-}\left(X^{-1}\right), \mathfrak{a}\right\}$ makes an $\mathfrak{s l}(2, \mathbb{R})$ triple. Therefore, there is an embedding of $\operatorname{SL}(2, \mathbb{R})$ into $H$ that sends $\left[\begin{array}{ll}1 & 1 \\ 0 & 1\end{array}\right]$ to $\exp \left(\mathfrak{n}^{+}(X)\right)=u(X)$, $\left[\begin{array}{ll}1 & 0 \\ 1 & 1\end{array}\right]$ to $\exp \left(\mathfrak{n}^{-}\left(X^{-1}\right)\right)=u^{-}\left(X^{-1}\right)$, and $\left[\begin{array}{cc}e^{t} & 0 \\ 0 & e^{-t}\end{array}\right]$ to $\exp (t \mathfrak{a})=a(t)$. We call the image of this $\operatorname{SL}(2, \mathbb{R})$ embedding $\operatorname{SL}(2, X) \subset H$. Let us denote

$$
\sigma(X):=\left[\begin{array}{ll}
X^{-1} & -X
\end{array}\right] \in \mathrm{SL}(2, X) .
$$

In this $\mathrm{SL}(2, \mathbb{R})$ copy, $\sigma(X)$ corresponds to $\left[\begin{array}{cc}0 & -1 \\ 1 & 0\end{array}\right] \in \mathrm{SL}(2, \mathbb{R})$.

2.2. Unipotent invariance of limit measures. Recall that for $t>0, \mu_{t}$ denotes the normalized Lebesgue measure on the curve $a(t) u(\varphi(I)) x$, and $\mu_{G}$ denotes the unique $G$ invariant probability measure on $G / \Gamma$. Our aim is to prove that $\mu_{t} \rightarrow \mu_{G}$ as $t \rightarrow+\infty$. We first modify the measures $\mu_{t}$ to another measure $\lambda_{t}$ and show that if $\lambda_{t} \rightarrow \mu_{G}$, then $\mu_{t} \rightarrow \mu_{G}$. Then we could study $\left\{\lambda_{t}: t>0\right\}$ instead. The motivation for this modification is that any accumulation point of $\left\{\lambda_{t}: t>0\right\}$ is invariant under a unipotent subgroup.

The measure $\lambda_{t}$ is defined as follows:

Definition 2.1. For $t>0$ and a closed subinterval $J \subset I$, suppose that the derivative $\varphi^{(1)}(s) \in \mathrm{GL}(n, \mathbb{R})$ for all $s \in J$. We define an analytic curve $z: J \rightarrow$ $Z_{H}(A)$ such that $\operatorname{Ad}_{z(s)}^{+}\left(\varphi^{(1)}(s)\right)=\mathrm{I}_{n}$ for all $s \in J$. Then we define $\lambda_{t}^{J}$ to be the normalized Lebesgue measure on $\{z(s) a(t) u(\varphi(s)) x: s \in J\}$, i.e., for $f \in C_{c}(G / \Gamma)$,

$$
\int f \mathrm{~d} \lambda_{t}^{J}:=\frac{1}{|J|} \int_{s \in J} f(z(s) a(t) u(\varphi(s)) x) \mathrm{d} s .
$$

Remark 2.2. Definition 2.1] is taken from [Sha09c] and [Sha09b].

For a subinterval $J \subset I$, we similarly define $\mu_{t}^{J}$ to be the normalized Lebesgue measure on $a(t) u(\varphi(J)) x$.

Proposition 2.3. Suppose for any closed subinterval $J \subset I$ such that $\lambda_{t}^{J}$ is defined, $\lambda_{t}^{J} \rightarrow \mu_{G}$ as $t \rightarrow+\infty$. Then $\mu_{t} \rightarrow \mu_{G}$ as $t \rightarrow+\infty$.

Proof. Let $s_{1}, s_{2}, \ldots, s_{l} \in I$ be all the points where $\varphi^{(1)}(s)$ is not invertible. For any fixed $f \in C_{c}(G / \Gamma)$ and $\epsilon>0$, we want to show that for $t>0$ large enough,

$$
\int f \mathrm{~d} \mu_{t} \stackrel{4 \epsilon}{\approx} \int_{G / \Gamma} f \mathrm{~d} \mu_{G}
$$


For each $i \in\{1,2, \ldots, l\}$, one could choose an open subinterval $B_{i} \subset I$ containing $s_{i}$, such that

$$
\left|\left(\sum_{i=1}^{l}\left|B_{i}\right|\right) \int_{G / \Gamma} f \mathrm{~d} \mu_{G}\right| \leq \epsilon|I|
$$

and for any $t>0$,

$$
\left|\int_{\bigcup_{i=1}^{l} B_{i}} f(a(t) u(\varphi(s)) x) \mathrm{d} s\right| \leq \epsilon|I| .
$$

Since $f$ is uniformly continuous, there exists a constant $\delta>0$, such that if $x_{1} \stackrel{\delta}{\approx} x_{2}$, then $f\left(x_{1}\right) \stackrel{\epsilon}{\approx} f\left(x_{2}\right)$.

We cut $I \backslash \bigcup_{i=1}^{l} B_{i}$ into several small closed subintervals $J_{1}, J_{2}, \ldots, J_{p}$, such that, for every $J_{r}, z^{-1}\left(s_{1}\right) z\left(s_{2}\right) \stackrel{\delta}{\approx} e$ for any $s_{1}, s_{2} \in J_{r}$.

Now for a fixed $J_{r} \subset I \backslash \bigcup_{i=1}^{l} B_{i}$, we choose $s_{0} \in J_{r}$ and define $f_{0}(x)=$ $f\left(z^{-1}\left(s_{0}\right) x\right)$. Then for any $s \in J_{r}$, because $z^{-1}\left(s_{0}\right) z(s) a(t) u(\varphi(s)) x \stackrel{\delta}{\approx} a(t) u(\varphi(s)) x$, we have

$$
f_{0}(z(s) a(t) u(\varphi(s)) x)=f\left(z^{-1}\left(s_{0}\right) z(s) a(t) u(\varphi(s)) x\right) \stackrel{\epsilon}{\approx} f(a(t) u(\varphi(s)) x) .
$$

Therefore

$$
\int f_{0} \mathrm{~d} \lambda_{t}^{J_{r}} \stackrel{\epsilon}{\approx} \int f \mathrm{~d} \mu_{t}^{J_{r}}
$$

Because $\int f_{0} \mathrm{~d} \lambda_{t}^{J_{r}} \rightarrow \int_{G / \Gamma} f_{0}(x) \mathrm{d} \mu_{G}(x)$ as $t \rightarrow+\infty$, and $\int_{G / \Gamma} f_{0}(x) \mathrm{d} \mu_{G}(x)=$ $\int_{G / \Gamma} f\left(z^{-1}\left(s_{0}\right) x\right) \mathrm{d} \mu_{G}(x)=\int_{G / \Gamma} f(x) \mathrm{d} \mu_{G}$ (because $\mu_{G}$ is $G$-invariant), we have that there exists a constant $T_{r}>0$, such that for $t>T_{r}$,

$$
\int f_{0} \mathrm{~d} \lambda_{t}^{J_{r}} \approx \int_{G / \Gamma} f \mathrm{~d} \mu_{G}
$$

Therefore, for $t>T_{r}$,

$$
\int f \mathrm{~d} \mu_{t}^{J_{r}} \stackrel{2 \epsilon}{\approx} \int_{G / \Gamma} f \mathrm{~d} \mu_{G}
$$

i.e.,

$$
\int_{J_{r}} f(a(t) u(\varphi(s)) x) \mathrm{d} s \stackrel{2 \epsilon\left|J_{r}\right|}{\approx}\left|J_{r}\right| \int_{G / \Gamma} f \mathrm{~d} \mu_{G}
$$

Then for $t>\max _{1 \leq r \leq p} T_{r}$, we could sum up the above approximations for $r=$ $1,2, \ldots, p$ and get

$$
\int_{I \backslash \bigcup_{i=1}^{r} B_{i}} f(a(t) u(\varphi(s)) x) \mathrm{d} s \stackrel{2 \epsilon|I|}{\approx}\left(|I|-\sum_{i=1}^{l}\left|B_{i}\right|\right) \int_{G / \Gamma} f \mathrm{~d} \mu_{G} .
$$

Combined with (2.1) and (2.2), the above approximation implies that

$$
\int_{I} f(a(t) u(\varphi(s)) x) \mathrm{d} s \stackrel{4 \epsilon|I|}{\approx}|I| \int_{G / \Gamma} f \mathrm{~d} \mu_{G},
$$

which is equivalent to

$$
\int f \mathrm{~d} \mu_{t} \stackrel{4 \epsilon}{\approx} \int_{G / \Gamma} f \mathrm{~d} \mu_{G}
$$

Because $\epsilon>0$ can be arbitrarily small, the proof is completed. 
By Proposition 2.3, to prove $\mu_{t} \rightarrow \mu_{G}$ as $t \rightarrow+\infty$, it suffices to show that for any closed subinterval $J \subset I$ where $\lambda_{t}^{J}$ is defined, $\lambda_{t}^{J} \rightarrow \mu_{G}$ as $t \rightarrow+\infty$. In particular, if we could prove the equidistribution of $\left\{\lambda_{t}:=\lambda_{t}^{I}: t>0\right\}$ as $t \rightarrow+\infty$ assuming $\varphi^{(1)}(s)$ is invertible for all $s \in I$, then the equidistribution of $\left\{\mu_{t}: t>0\right\}$ as $t \rightarrow+\infty$ will follow. Therefore, later in this paper, we will assume that $\varphi^{(1)}(s)$ is invertible for all $s \in I$ and define $\lambda_{t}$ to be the normalized Lebesgue measure on the curve $\{z(s) a(t) u(\varphi(s)) x: s \in I\}$. Our goal is to show that $\lambda_{t} \rightarrow \mu_{G}$ as $t \rightarrow+\infty$.

We will show that any limit measure of $\left\{\lambda_{t}: t>0\right\}$ is invariant under the unipotent subgroup $W=\left\{u\left(t \mathrm{I}_{n}\right): t \in \mathbb{R}\right\}$.

Proposition 2.4 (see Sha09c]). Let $t_{i} \rightarrow+\infty$ be a sequence such that $\lambda_{t_{i}} \rightarrow \mu_{\infty}$ in weak-* topology; then $\mu_{\infty}$ is invariant under the $W$-action.

Proof. Given any $f \in C_{c}(G / \Gamma)$, and $r \in \mathbb{R}$, we want to show that

$$
\int f\left(u\left(r \mathrm{I}_{n}\right) x\right) \mathrm{d} \mu_{\infty}=\int f(x) \mathrm{d} \mu_{\infty} .
$$

Since $z(s)$ and $\varphi(s)$ are analytic and defined on closed interval $I=[a, b]$, there exists a constant $T_{1}>0$ such that for $t \geq T_{1}, z(s)$ and $\varphi(s)$ can be extended to analytic curves defined on $\left[a-|r| e^{-2 t}, b+|r| e^{-2 t}\right]$. Throughout the proof, we always assume that $t_{i} \geq T_{0}$. Then $z\left(s+r e^{-2 t_{i}}\right)$ and $\varphi\left(s+r e^{-2 t_{i}}\right)$ are both well defined for $s \in I$.

From the definition of $\mu_{\infty}$ we have

$$
\int f\left(u\left(r \mathrm{I}_{n}\right) x\right) \mathrm{d} \mu_{\infty}=\lim _{t_{i} \rightarrow+\infty} \frac{1}{|I|} \int_{s \in I} f\left(u\left(r \mathrm{I}_{n}\right) z(s) a\left(t_{i}\right) u(\varphi(s)) x\right) \mathrm{d} s .
$$

We want to argue that

$$
u\left(r \mathrm{I}_{n}\right) z(s) a\left(t_{i}\right) u(\varphi(s)) \stackrel{O\left(e^{-2 t_{i}}\right)}{\approx} z\left(s+r e^{-2 t_{i}}\right) a\left(t_{i}\right) u\left(\varphi\left(s+r e^{-2 t_{i}}\right)\right) .
$$

Since $z\left(s+r e^{-2 t_{i}}\right) \stackrel{O\left(e^{-2 t_{i}}\right)}{\approx} z(s)$ for $t_{i}$ large enough, it suffices to show that

$$
u\left(r \mathrm{I}_{n}\right) z(s) a\left(t_{i}\right) u(\varphi(s)) \stackrel{O\left(e^{-2 t_{i}}\right)}{\approx} z(s) a\left(t_{i}\right) u\left(\varphi\left(s+r e^{-2 t_{i}}\right)\right) .
$$

In fact,

$$
\begin{aligned}
& z(s) a\left(t_{i}\right) u\left(\varphi\left(s+r e^{-2 t_{i}}\right)\right) \\
= & z(s) a\left(t_{i}\right) u\left(\varphi(s)+r e^{-2 t_{i}} \varphi^{\prime}(s)+O\left(e^{-4 t_{i}}\right)\right) \\
= & z(s) u\left(r \varphi^{\prime}(s)\right) u\left(O\left(e^{-2 t_{i}}\right)\right) a\left(t_{i}\right) u(\varphi(s)) .
\end{aligned}
$$

By the definition of $z(s)$, we have the above is equal to

$$
u\left(O\left(e^{-2 t_{i}}\right)\right) u\left(r \mathrm{I}_{n}\right) z(s) a\left(t_{i}\right) u(\varphi(s)) .
$$

This shows that

$$
u\left(r \mathrm{I}_{n}\right) z(s) a\left(t_{i}\right) u(\varphi(s)) \stackrel{O\left(e^{-2 t_{i}}\right)}{\approx} z\left(s+r e^{-2 t_{i}}\right) a\left(t_{i}\right) u\left(\varphi\left(s+r e^{-2 t_{i}}\right)\right) .
$$

Therefore, for any $\delta>0$, there is some constant $T \geq T_{1}$, such that for $t_{i} \geq T$,

$$
u\left(r \mathrm{I}_{n}\right) z(s) a\left(t_{i}\right) u(\varphi(s)) \stackrel{\delta}{\approx} z\left(s+r e^{-2 t_{i}}\right) a\left(t_{i}\right) u\left(\varphi\left(s+r e^{-2 t_{i}}\right)\right) .
$$

Given $\epsilon>0$, we choose $\delta>0$ such that whenever $x_{1} \stackrel{\delta}{\approx} x_{2}$, we have $f\left(x_{1}\right) \stackrel{\epsilon}{\approx}$ $f\left(x_{2}\right)$. Let $T>0$ be the constant as above. Then from the above argument, for $t_{i}>T$, we have

$$
f\left(u\left(r \mathrm{I}_{n}\right) z(s) a\left(t_{i}\right) u(\varphi(s)) x\right) \stackrel{\epsilon}{\approx} f\left(z\left(s+r e^{-2 t_{i}}\right) a\left(t_{i}\right) u\left(\varphi\left(s+r e^{-2 t_{i}}\right)\right) x\right),
$$


therefore,

$$
\begin{aligned}
& \frac{1}{|I|} \int_{s \in I} f\left(u\left(r \mathrm{I}_{n}\right) z(s) a\left(t_{i}\right) u(\varphi(s)) x\right) \mathrm{d} s \\
\approx & \frac{1}{|I|} \int_{s \in I} f\left(z\left(s+r e^{-2 t_{i}}\right) a\left(t_{i}\right) u\left(\varphi\left(s+r e^{-2 t_{i}}\right)\right) x\right) \mathrm{d} s \\
= & \frac{1}{|I|} \int_{a+r e^{-2 t_{i}}}^{b+r t_{i}} f\left(z(s) a\left(t_{i}\right) u(\varphi(s)) x\right) \mathrm{d} s .
\end{aligned}
$$

It is easy to see that when $t_{i}$ is large enough,

$$
\frac{1}{|I|} \int_{a+r e^{-2 t_{i}}}^{b+r e^{-2 t_{i}}} f\left(z(s) a\left(t_{i}\right) u(\varphi(s)) x\right) \mathrm{d} s \stackrel{\epsilon}{\approx} \frac{1}{|I|} \int_{a}^{b} f\left(z(s) a\left(t_{i}\right) u(\varphi(s)) x\right) \mathrm{d} s .
$$

Therefore, for $t_{i}$ large enough,

$$
\int f\left(u\left(r \mathrm{I}_{n}\right) x\right) \mathrm{d} \lambda_{t_{i}} \stackrel{2 \epsilon}{\approx} \int f(x) \mathrm{d} \lambda_{t_{i}}
$$

Letting $t_{i} \rightarrow+\infty$, we have

$$
\int f\left(u\left(r \mathrm{I}_{n}\right) x\right) \mathrm{d} \mu_{\infty} \approx 2 \epsilon \int f(x) \mathrm{d} \mu_{\infty}
$$

Since the above approximation is true for arbitrary $\epsilon>0$, we have that $\mu_{\infty}$ is $W$-invariant.

2.3. Nondivergence of limit measures. We will prove that any limit measure $\mu_{\infty}$ of $\left\{\lambda_{t}: t>0\right\}$ is still a probability measure of $G / \Gamma$.

To show the nondivergence of limit measures, it suffices to prove the following proposition:

Proposition 2.5 (see $[$ Sha09c]). For any $\epsilon>0$, there exists a compact subset $\mathcal{K}_{\epsilon} \subset G / \Gamma$ such that $\lambda_{t}\left(\mathcal{K}_{\epsilon}\right) \geq 1-\epsilon$ for all $t>0$.

The proof of the proposition is due to Nimish Shah Sha09c. Here we just modify the proof to fit our needs.

Definition 2.6. Let $\mathfrak{g}$ denote the Lie algebra of $G$, and let $d=\operatorname{dim} G$. We define

$$
\mathcal{V}:=\bigoplus_{i=1}^{d} \bigwedge^{i} \mathfrak{g}
$$

and fix a norm $\|\cdot\|$ on $\mathcal{V}$. Let $G$ act on $\mathcal{V}$ via $\bigoplus_{i=1}^{d} \bigwedge^{i} \operatorname{Ad}(\cdot)$. This defines a linear representation of $G$ :

$$
G \rightarrow \mathrm{GL}(\mathcal{V}) .
$$

The following theorem due to Kleinbock and Margulis [KM98] is the basic tool to prove the proposition:

Theorem 2.7 (see Dan84 and KM98). Fix a norm $\|\cdot\|$ on $\mathcal{V}$. There exist finitely many vectors $v_{1}, v_{2}, \ldots, v_{r} \in \mathcal{V}$ such that for each $i=1,2, \ldots, r$, the orbit $\Gamma v_{i}$ is discrete, and the following holds: for any $\epsilon>0$ and $R>0$, there exists a 
compact set $K \subset G / \Gamma$ such that for any $t>0$ and any subinterval $J \subset I$, one of the following holds:

S.1 There exist $\gamma \in \Gamma$ and $j \in\{1, \ldots, r\}$ such that

$$
\sup _{s \in J}\left\|a(t) u(\varphi(s)) g \gamma v_{j}\right\|<R \text {. }
$$

S.2

$$
|\{s \in J: a(t) u(\varphi(s)) x \in K\}| \geq(1-\epsilon)|J| .
$$

Remark 2.8. The proof for polynomial curves is due to Dani [Dan84, and the proof for analytic curves is due to Kleinbock and Margulis [KM98]. The crucial part to prove the above theorem is to find constants $C>0$ and $\alpha>0$ such that in this particular representation, all the coordinate functions of $a(t) u(\varphi(\cdot))$ are $(C, \alpha)$ good. Here a function $f: I \rightarrow \mathbb{R}$ is called $(C, \alpha)$-good if for any subinterval $J \subset I$ and any $\epsilon>0$, the following holds:

$$
|\{s \in J:|f(s)|<\epsilon\}| \leq C\left(\frac{\epsilon}{\sup _{s \in J}|f(s)|}\right)^{\alpha}|J| .
$$

The following basic lemma on representations of $\operatorname{SL}(2, \mathbb{R})$ due to Nimish Shah is crucial in the proof:

Lemma 2.9 (see $[$ Sha09c] $)$. Let $V$ be a representation of $\mathrm{SL}(2, \mathbb{R})$, fix a norm $\|\cdot\|$ on $V$. We define

$$
A=\left\{a(t)=\left[\begin{array}{ll}
e^{t} & \\
& e^{-t}
\end{array}\right]: t \in \mathbb{R}\right\}
$$

and

$$
U^{+}(A)=\left\{u(t)=\left[\begin{array}{ll}
1 & t \\
0 & 1
\end{array}\right]: t \in \mathbb{R}\right\} .
$$

Then for any $t>0$, there exists a constant $\kappa=\kappa(t)>0$ such that for any $v \in V$,

$$
\max \left\{\left\|v^{+}(A)\right\|,\left\|(u(t) v)^{+0}(A)\right\|\right\} \geq \kappa\|v\| .
$$

In $H=\mathrm{SL}(2 n, \mathbb{R})$, for any $X \in \mathrm{GL}(n, \mathbb{R}), u(X) \in \mathrm{SL}(2, X) \subset H$ corresponds to $\left[\begin{array}{ll}1 & 1 \\ 0 & 1\end{array}\right]$ in $\mathrm{SL}(2, \mathbb{R})$, where $\mathrm{SL}(2, X) \cong \mathrm{SL}(2, \mathbb{R})$ is defined in $\$ 2.1$. Lemma 2.9 easily implies the following:

Corollary 2.10 (see $[$ Sha09c] $)$. Let $V$ be a linear representation of $H=\mathrm{SL}(2 n, \mathbb{R})$. Fix a norm $\|\cdot\|$ on $V$. Let $A=\{a(t): t \in \mathbb{R}\} \subset H$ be the one-parameter diagonal subgroup defined in (1.1). Then for any $X \in \mathrm{GL}(n, \mathbb{R})$, there exists a constant $\kappa>0$ such that for any $v \in V$,

$$
\max \left\{\left\|v^{+}(A)\right\|,\left\|(u(X) v)^{+0}(A)\right\|\right\} \geq \kappa\|v\| .
$$

In particular, for any $t>0$, and any $v \in V$,

$$
\max \{\|a(t) v\|,\|a(t) u(X) v\|\} \geq \kappa\|v\| .
$$

Proof of Proposition 2.5. Fix $s_{0} \in I$ and a closed subinterval $J_{s_{0}}$ such that $\varphi(s)-$ $\varphi\left(s_{0}\right) \subset \mathrm{GL}(n, \mathbb{R})$ for $s \in J_{s_{0}}$. Let us fix $s_{1} \in J_{s_{0}}$. Let $\kappa>0$ be the constant given in Corollary 2.10 with $X$ replaced by $\varphi\left(s_{1}\right)-\varphi\left(s_{0}\right)$. 
For any $\epsilon>0$ and $R>0$, by Theorem 2.7. there exists a compact subset $K \subset G / \Gamma$, such that for any $t>0$, one of the following holds:

S.1 There exist $\gamma \in \Gamma$ and $j \in\{1, \ldots, r\}$ such that

$$
\sup _{s \in I}\left\|a(t) u(\varphi(s)) g \gamma v_{j}\right\|<R \text {. }
$$

S.2

$$
|\{s \in I: a(t) u(\varphi(s)) x \in K\}| \geq(1-\epsilon)|I| .
$$

Then because $\Gamma v_{i}$ is discrete in $\mathcal{V}$, there exists a uniform constant $r>0$ such that

$$
\left\|u\left(\varphi\left(s_{0}\right)\right) g \gamma v_{i}\right\| \geq r
$$

for any $v_{i}$ and $\gamma \in \Gamma$. Applying Corollary 2.10 with $v$ replaced by $u\left(\varphi\left(s_{0}\right)\right) g \gamma v_{i}$ and $X$ replaced by $\varphi\left(s_{1}\right)-\varphi\left(s_{0}\right)$, we get that for any $v_{i}, \gamma \in \Gamma$ and $t>0$,

$$
\sup _{s \in I}\left\|a(t) u(\varphi(s)) g \gamma v_{i}\right\| \geq \max \left\{a(t) u\left(\varphi\left(s_{0}\right)\right) g \gamma v_{i}, a(t) u\left(\varphi\left(s_{1}\right)\right) g \gamma v_{i}\right\} \geq \kappa r .
$$

If we choose $R<\kappa r$, then case $\mathbf{S . 1}$ above cannot hold. This shows that

$$
|\{s \in I: a(t) u(\varphi(s)) x \in K\}| \geq(1-\epsilon)|I| .
$$

Let $M$ be a compact subset of $Z_{H}(A)$ containing $z(I)$. Define $\mathcal{K}_{\epsilon}:=M K$. Since $z(s) \in M$, we have that

$$
\left|\left\{s \in I: z(s) a(t) u(\varphi(s)) x \in \mathcal{K}_{\epsilon}\right\}\right| \geq(1-\epsilon)|I|,
$$

i.e., $\lambda_{t}\left(\mathcal{K}_{\epsilon}\right) \geq 1-\epsilon$ for all $t>0$.

This completes the proof.

\section{RATNeR's TheOREM AND THE LINEARIZATION TECHNIQUE}

Take any convergent subsequence $\lambda_{t_{i}} \rightarrow \mu_{\infty}$. By Proposition 2.4 and Proposition 2.5. $\mu_{\infty}$ is a $W$-invariant probability measure on $G / \Gamma$.

In order to apply Ratner's Theorem and the linearization technique, we first introduce some notation.

Definition 3.1. Let $\mathcal{L}$ be the collection of analytic subgroups $L<G$ such that $L \cap \Gamma$ is a lattice of $L$. One could prove that $\mathcal{L}$ is a countable set (see Rat91a]).

For $L \in \mathcal{L}$, let us define

$$
N(L, W):=\left\{g \in G: g^{-1} W g \subset L\right\},
$$

and

$$
S(L, W):=\bigcup_{L^{\prime} \in \mathcal{L}, L^{\prime} \subsetneq L} N\left(L^{\prime}, W\right) .
$$

We recall Ratner's Measure Classification Theorem as follows:

Theorem 3.2 (see Rat91a). Recall that $\pi$ denotes the projection from $G$ to $X=$ $G / \Gamma$. Given the $W$-invariant probability measure $\mu$ on $G / \Gamma$, there exists $L \in \mathcal{L}$ such that

$$
\mu(\pi(N(L, W)))>0 \quad \text { and } \quad \mu(\pi(S(L, W)))=0 .
$$

Moreover, almost every $W$-ergodic component of $\mu$ on $\pi(N(L, W))$ is a measure of the form $g \mu_{L}$ where $g \in N(L, W) \backslash S(L, W), \mu_{L}$ is a finite L-invariant measure on $\pi(L)$, and $g \mu_{L}(E)=\mu_{L}\left(g^{-1} E\right)$ for all Borel sets $E \subset G / \Gamma$. In particular, if $L \triangleleft G$, then the restriction of $\mu$ to $\pi(N(L, W))$ is $L$-invariant. 
If $\mu_{\infty}=\mu_{G}$, then there is nothing to prove. So we assume $\mu_{\infty} \neq \mu_{G}$. By Ratner's Theorem, there exists $L \in \mathcal{L}, L \subsetneq G$, such that $\mu_{\infty}(\pi(N(L, W)))>0$ and $\mu_{\infty}(\pi(S(L, W)))=0$. Now we apply the linearization technique.

Definition 3.3. Let $\mathcal{V}$ be the finite dimensional representation of $G$ defined as in Definition 2.6. A subset $\mathcal{C} \subset \mathcal{V}$ is called symmetric if $\mathcal{C}=-\mathcal{C}$. For $L \in \mathcal{L}$, we choose a basis $\mathfrak{e}_{1}, \mathfrak{e}_{2}, \ldots, \mathfrak{e}_{l}$ of the Lie algebra $\mathfrak{l}$ of $L$, and define

$$
p_{L}=\bigwedge_{i=1}^{l} \mathfrak{e}_{i} \in \mathcal{V} .
$$

Let $N_{G}(L)$ denote the normalizer of $L$ in $G$. It is easy to see that

$$
N_{G}(L)=\left\{g \in G: g p_{L} \in \mathbb{R} \cdot p_{L}\right\} .
$$

Define

$$
N_{G}^{1}(L):=\left\{g \in G: g p_{L}=p_{L}\right\}
$$

and

$$
\Gamma_{L}:=\left\{\gamma \in \Gamma: \gamma p_{L}= \pm p_{L}\right\}
$$

The action of $G$ on $p_{L}$ defines a map:

$$
\begin{aligned}
& \eta: G \rightarrow \mathcal{V}, \\
& g \mapsto g p_{L} .
\end{aligned}
$$

We define $\mathcal{A}$ to be the Zariski closure of $\eta(N(L, W))$. For any compact subset $\mathcal{D} \subset \mathcal{A}$, we define

$$
S(\mathcal{D}):=\left\{g \in N(L, W): \eta(g \gamma) \in \mathcal{D} \text { for some } \gamma \in \Gamma \backslash \Gamma_{L}\right\} .
$$

We need the following propositions:

Proposition 3.4 (see Sha09c], Proposition 4.5). $S(\mathcal{D}) \subset S(L, W)$ and $\pi(S(\mathcal{D})$ ) is closed in $G / \Gamma$. Moreover, for any compact set $\mathcal{K} \in G / \Gamma \backslash \pi(S(\mathcal{D}))$, there exists a neighborhood $\Phi$ of $\mathcal{D}$ in $\mathcal{V}$ such that, for any $g \in G$ and $\gamma_{1}, \gamma_{2} \in \Gamma$, if $\pi(g) \in \mathcal{K}$ and $\eta\left(g \gamma_{i}\right) \in \Phi, i=1,2$, then $\eta\left(\gamma_{1}\right)= \pm \eta\left(\gamma_{2}\right)$.

Proposition 3.5 (see Sha09c, Proposition 4.6). Given a symmetric compact subset $\mathcal{C} \subset \mathcal{A}$ and $\epsilon>0$, there exists a symmetric compact set $\mathcal{D} \subset \mathcal{A}$ containing $\mathcal{C}$ such that, given a symmetric neighborhood $\Phi$ of $\mathcal{D}$ in $\mathcal{V}$, there exists a symmetric neighborhood $\Psi$ of $\mathcal{C}$ in $\mathcal{V}$ contained in $\Phi$ such that for any $t>0$, for any $v \in \mathcal{V}$, and for any interval $J \subset I$, one of the following holds:

SS.1 $a(t) u(\varphi(s)) v \in \Phi$ for all $s \in J$.

SS.2 $|\{s \in J: a(t) u(\varphi(s)) v \in \Psi\}| \leq \epsilon|\{s \in J: a(t) u(\varphi(s)) v \in \Phi\}|$.

Remark 3.6. The proof is similar to Theorem 2.7, and also follows from the fact that all coordinate functions of $a(t) u(\varphi(\cdot)) v$ are $(C, \alpha)$-good for some constants $C>0$ and $\alpha>0$.

The following proposition is the aim of this section.

Proposition 3.7. There exists $a \gamma \in \Gamma$ such that

$$
u(\varphi(s)) g \gamma p_{L} \in \mathcal{V}^{-0}(A),
$$

for all $s \in I$. 
Proof. Take a compact subset $C \subset N(L, W)) \backslash S(L, W)$ such that $\mu_{\infty}(\pi(C))>c_{0}>$ 0 for some constant $c_{0}$. Define $\mathcal{C}:=\eta(C) \cup(-\eta(C))$; then $\mathcal{C} \subset \mathcal{A}$ is a symmetric compact subset. Choose a compact subset $\mathcal{K} \subset G / \Gamma \backslash \pi(S(L, W))$ containing $\pi(C)$ in its interior. Applying Proposition 3.5] we can find a symmetric compact subset $\mathcal{D} \subset \mathcal{A}$ containing $\mathcal{C}$ such that the conclusion of Proposition 3.5 holds for $\mathcal{C}, \mathcal{D}$ and some small $0<\epsilon<\frac{c_{0}}{2}$. Applying Proposition 3.4 to $\mathcal{D}$ and $\mathcal{K}$, we have that there exists an open neighborhood $\Phi$ of $\mathcal{D}$ such that the conclusion of Proposition 3.4 holds. Choose a neighborhood $\Psi$ of $\mathcal{C}$ according to Proposition 3.5

We claim that for any $i \in \mathbb{N}$ large enough, there exists $\gamma_{i} \in \Gamma$ such that

$$
a\left(t_{i}\right) u(\varphi(I)) g \gamma_{i} p_{L} \subset \Phi .
$$

For contradiction, we assume that it is not the case, i.e., there exists an infinite subsequence of $\mathbb{N}$ such that for any $i$ in this subsequence, and any $\gamma \in \Gamma$, $a\left(t_{i}\right) u(\varphi(I)) g \gamma p_{L}$ is not contained in $\Phi$. This implies that case SS.1 in Proposition 3.5 does not hold for $v=g \gamma p_{L}$ and $J=I$. We define

$$
J_{i}:=\left\{s \in I: a\left(t_{i}\right) u(\varphi(s)) x \in \mathcal{K}: a\left(t_{i}\right) u(\varphi(s)) g \Gamma p_{L} \cap \Psi \neq \emptyset\right\} .
$$

Since $\mu_{\infty}(\pi(C))>c_{0}$, we have that for $i$ large enough, $\left|J_{i}\right|>c_{0}|I|$.

By Proposition 3.4 for any $s \in J_{i}$, up to \pm sign, there exists unique $\gamma(s) p_{L}$ such that $a\left(t_{i}\right) u(\varphi(s)) g \gamma(s) p_{L} \in \Psi$. Let $I_{\gamma(s)}$ be the maximal interval $I$ containing $s$ such that

$$
a\left(t_{i}\right) u\left(\varphi\left(I_{\gamma(s)}\right)\right) g \gamma(s) p_{L} \subset \Phi .
$$

By Proposition 3.4, there is no other $\gamma^{\prime} p_{L}$ other than $\pm \gamma(s) p_{L}$ and $s \in I_{\gamma(s)} \cap J_{i}$ such that

$$
a\left(t_{i}\right) u(\varphi(s)) g \gamma^{\prime} p_{L} \in \Psi .
$$

Therefore $J_{i}$ is covered by at most countably many intervals $I_{\gamma(s)}$ 's which covers the whole interval $I$ at most twice, namely, every point belongs to at most two different intervals (this is because for any $s_{1}<s_{2} \in J_{i}, I_{\gamma\left(s_{1}\right)} \cap I_{\gamma\left(s_{2}\right)} \subset\left(s_{1}, s_{2}\right)$ ). Because case SS.1 in Proposition 3.5 does not hold, we have that SS.2 must hold, i.e.,

This shows that

$$
\left|J_{i} \cap I_{\gamma(s)}\right|<\epsilon\left|I_{\gamma(s)}\right|
$$

$$
\left|J_{i}\right|<2 \epsilon|I| \text {. }
$$

This contradicts the fact that $\left|J_{i}\right|>c_{0}|I|$. This shows the claim.

Since $\Gamma p_{L}$ is discrete in $\mathcal{V}$, one of the following will happen:

(1) $\left\|\gamma_{i} p_{L}\right\| \rightarrow+\infty$ as $i \rightarrow \infty$.

(2) $\gamma_{i} p_{L}$ remains the same for all large $i$.

If the first case happens, we define a unit vector $v_{i}=\frac{\gamma_{i} p_{L}}{\left\|\gamma_{i} p_{L}\right\|}$ for each $i$. Because

$$
a\left(t_{i}\right) u(\varphi(I)) \gamma_{i} p_{L} \subset \Phi,
$$

there is a constant $R$ such that

$$
\sup _{s \in I}\left\|a\left(t_{i}\right) u(\varphi(s)) v_{i}\right\| \leq \frac{R}{\left\|\gamma_{i} p_{L}\right\|} \rightarrow 0 .
$$

Suppose $v_{i} \rightarrow v_{\infty}$ passing to some subsequence; then we have that

$$
\sup _{s \in I}\left\|a\left(t_{i}\right) u(\varphi(s)) v_{\infty}\right\| \rightarrow 0,
$$


as $i \rightarrow \infty$. This is impossible by Corollary 2.10. Therefore $\gamma_{i} p_{L}=\gamma p_{L}$ remains the same for all large $i$. This means that for all $i$ large enough,

$$
\sup _{s \in I}\left\|a\left(t_{i}\right) u(\varphi(s)) g \gamma p_{L}\right\| \leq R .
$$

This implies that for $v=g \gamma p_{L}$,

$$
u(\varphi(s)) v \in \mathcal{V}^{-0}(A) .
$$

This completes the proof.

\section{Conclusion}

In this section we will prove Theorem 1.4. By the correspondence between homogeneous dynamics and Diophantine approximation discussed in $\$ 1$ Theorem 1.2 follows from Theorem 1.4 .

We need the following basic lemma on $\mathrm{SL}(2, \mathbb{R})$ representations.

Lemma 4.1 (see Yan13, Lemma 5.1). Let $V$ be a finite dimensional linear representation of $\operatorname{SL}(2, \mathbb{R})$. Denote

$$
A:=\left\{a(t):=\left[\begin{array}{ll}
e^{t} & \\
& e^{-t}
\end{array}\right]: t \in \mathbb{R}\right\}
$$

and

$$
U:=\left\{u(s):=\left[\begin{array}{ll}
1 & s \\
0 & 1
\end{array}\right]\right\} .
$$

Suppose there is a nonzero vector $v \in V^{-0}(A)$ satisfying

$$
u(r) v \in V^{-0}(A),
$$

for some $r \in \mathbb{R}$; then $(u(r) v)^{0}(A)=\sigma v^{0}(A)$, where $\sigma$ denotes the matrix

$$
\sigma=\left[\begin{array}{cc}
0 & -1 \\
1 & 0
\end{array}\right]
$$

Proof of Theorem 1.4. We start with the linear algebraic condition we get from Proposition 3.7

$$
u(\varphi(s)) v \in \mathcal{V}^{-0}(A), \quad \text { for all } s \in I .
$$

We first prove the following claim:

Claim 4.2. $(u(\varphi(s)) v)^{0}(A)$ is invariant under the action of the unipotent subgroup $\left\{u\left(r \varphi^{(1)}(s)\right): r \in \mathbb{R}\right\}$.

Proof of the claim. On the one hand, since $u(\varphi(s)) v \in \mathcal{V}^{-0}(A)$ for all $s \in I$, for any fixed $r \in \mathbb{R}$,

$$
\lim _{t \rightarrow+\infty} a(t) u\left(\varphi\left(s+r e^{-2 t}\right)\right) v=\lim _{t \rightarrow+\infty}\left(u\left(\varphi\left(s+r e^{-2 t}\right)\right) v\right)^{0}(A)=(u(\varphi(s)) v)^{0}(A) .
$$

On the other hand,

$$
\begin{aligned}
& \lim _{t \rightarrow+\infty} a(t) u\left(\varphi\left(s+r e^{-2 t}\right)\right) v \\
= & \lim _{t \rightarrow+\infty} a(t) u\left(\varphi(s)+r \varphi^{(1)}(s) e^{-2 t}+O\left(e^{-4 t}\right)\right) v \\
= & \lim _{t \rightarrow+\infty} a(t) u\left(r \varphi^{(1)}(s) e^{-2 t}+O\left(e^{-4 t}\right)\right) a(-t) a(t) u(\varphi(s)) v \\
= & \lim _{t \rightarrow+\infty} u\left(r \varphi^{(1)}(s)+O\left(e^{-2 t}\right)\right) a(t) u(\varphi(s)) v \\
= & u\left(r \varphi^{(1)}(s)\right)(u(\varphi(s)) v)^{0}(A) .
\end{aligned}
$$

This implies $(u(\varphi(s)) v)^{0}(A)=u\left(r \varphi^{(1)}(s)\right)(u(\varphi(s)) v)^{0}(A)$ for all $r \in \mathbb{R}$.

This proves the claim. 
Fix $s_{0} \in I$ and a closed subinterval $J_{s_{0}} \subset I$ such that $\varphi^{(1)}\left(s_{0}\right)$ is invertible, and $\varphi(s)-\varphi\left(s_{0}\right)$ is invertible for all $s \in J_{s_{0}}$. For $s \in J_{s_{0}}$, let us denote $X(s)=$ $\varphi(s)-\varphi\left(s_{0}\right)$, and consider the subgroup $\mathrm{SL}(2, X(s)) \cong \mathrm{SL}(2, \mathbb{R})$. Note that in $\mathrm{SL}(2, X(s))$,

corresponds to

$$
\sigma(X(s))=\left[\begin{array}{ll}
X^{-1}(s) & -X(s)
\end{array}\right]
$$

$$
\sigma=\left[\begin{array}{cc}
0 & -1 \\
1 & 0
\end{array}\right] \in \mathrm{SL}(2, \mathbb{R})
$$

Applying Lemma 4.1 with $\mathrm{SL}(2, \mathbb{R})$ replaced by $\mathrm{SL}(2, X(s)), v$ replaced by $u\left(\varphi\left(s_{0}\right)\right) v$ and $u(r)$ replaced by $u(X(s))$, we conclude that

$$
(u(\varphi(s)) v)^{0}(A)=\sigma(X(s))\left(u\left(\varphi\left(s_{0}\right)\right) v\right)^{0}(A) .
$$

Let us denote $w=\left(u\left(\varphi\left(s_{0}\right)\right) v\right)^{0}(A)$ and define

$$
\mathcal{S}:=\left\{X \in \mathrm{M}(n \times n, \mathbb{R}): u^{-}(X) w=w\right\} .
$$

It is easy to see that $\mathcal{S}$ is a subspace of $\mathrm{M}(n \times n, \mathbb{R})$.

Claim 4.3. $\mathcal{S}$ is a proper subspace of $\mathrm{M}(n \times n, \mathbb{R})$.

Proof of the claim. Suppose not, then $w$ is fixed by the whole horospherical subgroup $U^{-}(A) . w$ is also fixed by $A$ since it is in $\mathcal{V}^{0}(A)$. This implies that $w$ is fixed by $H=\operatorname{SL}(2 n, \mathbb{R})$. Let us denote $u\left(\varphi\left(s_{0}\right)\right) v=w+w^{-}$, where $w^{-}=u\left(\varphi\left(s_{0}\right)\right) v-w \in$ $\mathcal{V}^{-}(A)$. We claim that $w^{-}=\mathbf{0}$. In fact, if $w^{-} \neq \mathbf{0}$, then for $s \in J$,

$$
u(X(s)) w^{-}=u(\varphi(s)) v-u(X(s)) w=u(\varphi(s)) v-w \in \mathcal{V}^{-0}(A) .
$$

This contradicts Lemma 2.9 with $\operatorname{SL}(2, \mathbb{R})$ replaced by $\operatorname{SL}(2, X(s)), u(r)$ replaced by $u(X(s))$, and $v$ replaced by $w^{-}$. This shows that $w^{-}=\mathbf{0}$. Thus, $u\left(\varphi\left(s_{0}\right)\right) v=w$ is fixed by the whole group $H=\operatorname{SL}(2 n, \mathbb{R})$. Then $v=g \gamma p_{L}$ is fixed by $H$. Hence $p_{L}$ is fixed by the action of $\gamma^{-1} g^{-1} H g \gamma$. Thus

$$
\begin{aligned}
\Gamma p_{L} & =\overline{\Gamma p_{L}}\left(\text { since } \Gamma p_{L} \text { is discrete }\right) \\
& =\overline{\Gamma \gamma^{-1} g^{-1} H g \gamma p_{L}} \\
& =\overline{\Gamma g^{-1} H g \gamma p_{L}} \\
& =G g \gamma p_{L}(\text { since } \overline{H g \Gamma}=G) \\
& =G p_{L} .
\end{aligned}
$$

This implies that $G_{0} p_{L}=p_{L}$ where $G_{0}$ denotes the connected component of $e$ in $G$. In particular, $\gamma^{-1} g^{-1} H g \gamma \subset G_{0}$ and $G_{0} \subset N_{G}^{1}(L)$. By Sha09a, Theorem 2.3], there exists a closed subgroup $F_{1} \subset N_{G}^{1}(L)$ containing all Ad-unipotent oneparameter subgroups of $G$ contained in $N_{G}^{1}(L)$ such that $F_{1} \cap \Gamma$ is a lattice in $F_{1}$ and $\pi\left(F_{1}\right)$ is closed. If we put $F=g \gamma F_{1} \gamma^{-1} g^{-1}$, then $H \subset F$ since $H$ is generated by its unipotent one-parameter subgroups. Moreover, $F x=g \gamma \pi\left(F_{1}\right)$ is closed and admits a finite $F$-invariant measure. Then since $\overline{H x}=G / \Gamma$, we have $F=G$. This implies $F_{1}=G$ and thus $L \triangleleft G$. Therefore $N(L, W)=G$. In particular, $W \subset L$, and thus $L \cap H$ is a normal subgroup of $H$ containing $W$. Since $H$ is a simple group, we have that $H \subset L$. Since $L$ is a normal subgroup of $G$ and $\pi(L)$ is a closed orbit with finite $L$-invariant measure, every orbit of $L$ on $G / \Gamma$ is also closed and admits a finite $L$-invariant measure, in particular, $L x$ is closed. But since $H x$ is dense in $G / \Gamma, L x$ is also dense. This shows that $L=G$, which contradicts our hypothesis that $\mu_{\infty} \neq \mu_{G}$. This proves the claim. 
Let $\langle\cdot, \cdot\rangle$ denote the standard inner product on $\mathrm{M}(n \times n, \mathbb{R}) \cong \mathbb{R}^{n^{2}}$. Then there exists a nonzero vector $\mathbf{Y} \in \mathrm{M}(n \times n, \mathbb{R})$ such that any $X \in \mathcal{S}$ satisfies $\langle X, \mathbf{Y}\rangle=0$.

We have proved that $(u(\varphi(s)) v)^{0}(A)$ is fixed by $\left\{u\left(r \varphi^{(1)}(s)\right): r \in \mathbb{R}\right\}$. By (4.1), we have that $w=\left(u\left(\varphi\left(s_{0}\right)\right) v\right)^{0}(A)$ is fixed by

$$
(\sigma(X(s)))^{-1} u\left(\varphi^{(1)}\left(s_{0}\right)\right) \sigma(X(s))=\left[\begin{array}{cc}
\mathrm{I}_{n} & \\
H(s) & \mathrm{I}_{n}
\end{array}\right],
$$

where $H(s):=-X(s)^{-1} \varphi^{(1)}(s) X(s)^{-1}$. This means $\left\langle-X(s)^{-1} \varphi^{(1)}(s) X(s)^{-1}, \mathbf{Y}\right\rangle$ $=0$. Note that

$$
\left((X(s))^{-1}\right)^{(1)}=-X(s)^{-1} \varphi^{(1)}(s) X(s)^{-1} .
$$

This implies that $\left\langle(X(s))^{-1}, \mathbf{Y}\right\rangle$ is a constant, i.e., $\left\{X(s)^{-1}=\left(\varphi(s)-\varphi\left(s_{0}\right)\right)^{-1}\right.$ : $s \in J\}$ is contained in a proper affine subspace of $\mathrm{M}(n \times n, \mathbb{R})$ orthogonal to $\mathbf{Y}$. Because this holds for arbitrary $s_{0} \in I$, we get a contradiction to condition $\mathbf{A . 2}$ in Theorem 1.2

This completes the proof of Theorem 1.4

Remark 4.4. We call a proper subspace $\mathcal{S}$ of $\mathrm{M}(n \times n, \mathbb{R})$ observable if there exists a nonzero vector $v \in \mathcal{V}$ fixed by $A$ such that $\mathcal{S}=\left\{X \in \mathrm{M}(n \times n, \mathbb{R}): u^{-}(X) v=v\right\}$. A proper affine subspace of $\mathrm{M}(n \times n, \mathbb{R})$ is called observable if it is parallel to an observable subspace. From the proof we see that if the equidistribution does not hold, then $\left\{\left(\varphi(s)-\varphi\left(s_{0}\right)^{-1}: s \in J_{s_{0}}\right\}\right.$ must be contained in an observable affine subspace. Therefore, to ensure the equidistribution, we only need $\left\{\left(\varphi(s)-\varphi\left(s_{0}\right)^{-1}\right.\right.$ : $\left.s \in J_{s_{0}}\right\}$ to avoid observable affine subspaces of $\mathrm{M}(n \times n, \mathbb{R})$.

\section{Appendix A. Relation between the condition given in ABRdS14 And CONDITION A.1 IN ThEOREM 1.2}

We will discuss the condition given in ABRdS14 and its relation to condition A.1 in Theorem 1.2. Because in this paper we only consider the case $m=n$, we only discuss this special case.

We denote $M(s)=\left[\mathrm{I}_{n}, \varphi(s)\right] \in \mathrm{M}(n \times 2 n, \mathbb{R})$. Given a subspace $W \subset \mathbb{R}^{2 n}$ and $0<r<\frac{\operatorname{dim} W}{2}$, we define the pencil $\mathcal{P}_{W, r}$ as follows:

$$
\mathcal{P}_{W, r}:=\{M \in \mathrm{M}(n \times 2 n, \mathbb{R}): \operatorname{dim} M W=r\} .
$$

In ABRdS14, the following theorem is announced: if a submanifold is not contained in a pencil defined as above, then the submanifold is extremal. In our setup, it says that if the curve $\{M(s): s \in I\}$ is not contained in any pencil $\mathcal{P}_{W, r}$, then the curve is extremal. It is easy to see that if $W$ is a rational subspace, then $\mathcal{P}_{W, r}$ is not extremal. So this condition is almost optimal.

Proposition A.1. If the curve $\varphi(I)$ satisfies that for some $s_{0} \in I, \varphi(s)-\varphi\left(s_{0}\right)$ is invertible for $s$ in a subinterval $J$ of $I$, then the curve $\{M(s): s \in I\}$ is not contained in any pencil $\mathcal{P}_{W, r}$.

Proof. Suppose not; then the curve $\left\{\left[\mathrm{I}_{n}, \varphi(s)\right]: s \in I\right\}$ is contained in some pencil $\mathcal{P}_{W, r}$ where $r<\frac{\operatorname{dim} W}{2}$. This means that if we denote $M(s)=\left[\mathrm{I}_{n}, \varphi(s)\right]$, the intersection of $W$ and the kernel of $M(s)$ has dimension greater than $\frac{\operatorname{dim} W}{2}$, i.e.,

$$
\operatorname{dim} \operatorname{Ker}(M(s)) \cap W>\frac{\operatorname{dim} W}{2} .
$$


Let us denote $W(s):=\operatorname{Ker}(M(s)) \cap W$. Then for any $s_{1} \neq s_{2} \in I, W\left(s_{1}\right) \cap W\left(s_{2}\right) \neq$ $\{\mathbf{0}\}$ because the sum of their dimensions is greater than $\operatorname{dim} W$. This means that the intersection $\operatorname{Ker}\left(M\left(s_{1}\right)\right) \cap \operatorname{Ker}\left(M\left(s_{2}\right)\right) \neq\{\mathbf{0}\}$. It is easy to see that the kernel of $M(s)$ is $\left\{(-\varphi(s) w, w): w \in \mathbb{R}^{n}\right\}$, so there exist $w_{1}, w_{2} \in \mathbb{R}^{n} \backslash\{\mathbf{0}\}$ such that

$$
\left(-\varphi\left(s_{1}\right) w_{1}, w_{1}\right)=\left(-\varphi\left(s_{2}\right) w_{2}, w_{2}\right) .
$$

This implies that $w_{1}=w_{2}$, and $-\varphi\left(s_{1}\right) w_{1}=-\varphi\left(s_{2}\right) w_{2}$. This implies that $\left(\varphi\left(s_{1}\right)-\right.$ $\left.\varphi\left(s_{2}\right)\right) w_{1}=\mathbf{0}$ for nonzero vector $w_{1}$. This shows that $\varphi\left(s_{1}\right)-\varphi\left(s_{2}\right)$ is not invertible, for any $s_{1} \neq s_{2} \in I$. This gives a contradiction.

This shows that condition A.1 implies the condition given in ABRdS14].

\section{ACKNOWLEDGEMENT}

The author would like to express deep gratitude to his advisor, Professor Nimish Shah, for suggesting this problem, and for his continuous advice and support during the process of the work. The author also would like to thank Professor Kleinbock for reading an earlier version of this paper and providing many comments and suggestions. For example, he drew [KMW10] and ABRdS14 to the author's attention and suggested the problem of figuring out the relation between the conditions given in this paper and that in ABRdS14.

Thanks are due to the referees for valuable suggestions.

\section{REFERENCES}

[ABRdS14] Menny Aka, Emmanuel Breuillard, Lior Rosenzweig, and Nicolas de Saxcé. On metric diophantine approximation in matrices and lie groups. arXiv preprint arXiv:1410.3996, 2014.

[Bak78] R. C. Baker, Dirichlet's theorem on Diophantine approximation, Math. Proc. Cambridge Philos. Soc. 83 (1978), no. 1, 37-59. MR.0485713

[Bug02] Yann Bugeaud, Approximation by algebraic integers and Hausdorff dimension, J. London Math. Soc. (2) 65 (2002), no. 3, 547-559, DOI 10.1112/S0024610702003137. MR.1895732

[Dan84] S. G. Dani, On orbits of unipotent flows on homogeneous spaces, Ergodic Theory Dynam. Systems 4 (1984), no. 1, 25-34, DOI 10.1017/S0143385700002248. MR.758891

[DS70] H. Davenport and W. M. Schmidt, Dirichlet's theorem on diophantine approximation. II, Acta Arith. 16 (1969/1970), 413-424. MR0279040

[KM98] Dmitry Y. Kleinbock and Grigorij A. Margulis, Flows on homogeneous spaces and diophantine approximation on manifolds, Annals of mathematics, pages 339-360, 1998.

[KMW10] Dmitry Kleinbock, Gregory Margulis, and Junbo Wang, Metric Diophantine approximation for systems of linear forms via dynamics, Int. J. Number Theory 6 (2010), no. 5, 1139-1168, DOI 10.1142/S1793042110003423. MR2679461

[KW08] Dmitry Kleinbock and Barak Weiss, Dirichlet's theorem on Diophantine approximation and homogeneous flows, J. Mod. Dyn. 2 (2008), no. 1, 43-62. MR2366229

[Rat91a] Marina Ratner, On Raghunathan's measure conjecture, Ann. of Math. (2) 134 (1991), no. 3, 545-607, DOI 10.2307/2944357. MR1135878

[Rat91b] Marina Ratner, Raghunathan's topological conjecture and distributions of unipotent flows, Duke Math. J. 63 (1991), no. 1, 235-280, DOI 10.1215/S0012-7094-91-06311-8. MR.1106945

[Sha09a] Nimish A. Shah, Asymptotic evolution of smooth curves under geodesic flow on hyperbolic manifolds, Duke Math. J. 148 (2009), no. 2, 281-304, DOI 10.1215/001270942009-027. MR2524497

[Sha09b] Nimish A. Shah, Equidistribution of expanding translates of curves and Dirichlet's theorem on Diophantine approximation, Invent. Math. 177 (2009), no. 3, 509-532, DOI 10.1007/s00222-009-0186-6. MR2534098 
[Sha09c] Nimish A. Shah, Limiting distributions of curves under geodesic flow on hyperbolic manifolds, Duke Math. J. 148 (2009), no. 2, 251-279, DOI 10.1215/00127094-2009026. MR 2524496

[Yan13] Lei Yang, Expanding curves in $\mathrm{T}^{1}\left(\mathbb{H}^{n}\right)$ under geodesic flow and equidistribution in homogeneous spaces, arXiv preprint arXiv:1303.6023, 2013.

Mathematical Sciences Research Institute, Berkeley, California 94720

Current address: Einstein Institute of Mathematics, Hebrew University of Jerusalem, Jerusalem, 9190401, Israel

E-mail address: yang.lei@mail.huji.ac.il 